\title{
Potential prognostic value of $P D-L 1$ and NKG2A expression in Indonesian patients with skin nodular melanoma
}

\author{
Ridwan Dwi Saputro ${ }^{1}$, Hanggoro Tri Rinonce ${ }^{2^{*}}$ (D), Yayuk Iramawasita ${ }^{2}$, Muhammad Rasyid Ridho², \\ Maria Fransiska Pudjohartono ${ }^{2}$, Sumadi Lukman Anwar' ${ }^{1}$ Kunto Setiaji ${ }^{1}$ and Teguh Aryandono ${ }^{1}$
}

\begin{abstract}
Objective: Biomarker mRNA levels have been suggested to be predictors of patient survival and therapy response in melanoma cases. This study aimed to investigate the correlations between the mRNA expression levels of $P D-L 1$ and NKG2A in melanoma tissue with clinicopathologic characteristics and survival in Indonesian primary nodular melanoma patients.

Results: Thirty-one tissue samples were obtained; two were excluded from survival analysis due to Breslow depth of less than $4 \mathrm{~mm}$. The median survival of upregulated and normoregulated PD-L 1-patients were $15.800 \pm 2.345$ and $28.945 \pm 4.126$ months, respectively. However, this difference was not significant statistically $(p=0.086)$. Upregulated and normoregulated NKG2A patients differed very little in median survival time (25.943 \pm 7.415 vs $26.470 \pm 3.854$ months; $p=0.981)$. Expression of $P D-L 1$ and NKG2A were strongly correlated $\left(r_{s}: 0.787, p<0.001\right)$. No clinicopathologic associations with PD-L1 and NKG2A mRNA levels were observed. These results suggest that PD-L1 may have potential as a prognostic factor. Although an unlikely prognostic factor, NKG2A may become an adjunct target for therapy. The strong correlation between PD-L1 and NKG2A suggests that anti-PD-1 and anti-NKG2A agents could be effective in patients with $P D-L 1$ upregulation. The mRNA levels of these two genes may help direct choice of immunotherapy and predict patient outcomes.
\end{abstract}

Keywords: PD-L1, NKG2A, Melanoma, Skin cancer, Indonesia

\section{Introduction}

Among cutaneous malignancies, melanoma results in the highest mortality; statistics revealed that melanomas caused 287,723 new cases and 60,712 deaths worldwide in 2018 [1]. These numbers are expected to increase, with an estimated 340,721 new cases projected in 2025 [2]. Trials of various systemic chemotherapy combinations did not improve survival significantly. Thus, current

\footnotetext{
*Correspondence: hanggoro_rinonce@ugm.ac.id

${ }^{2}$ Department of Anatomical Pathology, Faculty of Medicine, Public Health and Nursing, Universitas Gadjah Mada/Dr. Sardjito Hospital, Sleman, Yogyakarta, Indonesia

Full list of author information is available at the end of the article
}

research is focused on new therapeutic agents, including immune checkpoint blockers.

Agents that block immune checkpoints, such as $P D$ $1 / P D-L 1$, help improve the immune response to cancerous cells. Nivolumab, an anti- $P D-1$ antibody, has been approved by the FDA for the treatment of advanced melanomas [3]. Other immunotherapies, such as monalizumab, a humanized anti-NKG2A antibody that enhances NK cell and CD8+ T cell activity [4], are also under development. These drugs show great promise and more durable responses compared with targeted therapy agents.

Before starting anti- $P D-1$ immunotherapy, clinicians commonly test tumor tissues for $P D-L 1$ expression by 
using immunohistochemistry (IHC). Tumors expressing $P D-L 1$ respond better to anti- $P D-1 / P D-L 1$ agents compared with non-expressers [5]. However, the results of recent studies on the effect of $P D-L 1$ expression on survival are conflicting [6]. Detection using IHC also presents several limitations, such as the varying performance of different antibodies, nonstandard cut-off values, and operator dependence $[7,8]$.

Researchers are exploring new methods to predict therapy responses and survival in melanoma patients. Several studies have investigated the use of biomarker mRNA levels as an alternative parameter [9-11]. Gupta et al. reported that mRNA levels of $P D-L 1 / 2$ show potential in predicting survival and response toward immunotherapy in metastatic melanoma [12]. Given the emergence of monalizumab, the potential prognostic and therapeutic roles of NKG2A should also be investigated.

Immuno-oncological research is still rare in Indonesia. Most Indonesian patients are treated with surgical resection, dacarbazine chemotherapy, and radiotherapy. Information on the expression of immune-checkpoint molecules is needed to gauge the potential efficacy of using immunotherapy agents in Indonesia. Thus, this study aimed to investigate the prognostic role of mRNA levels of $P D-L 1$ and $N K G 2 A$, as well as the associated clinicopathologic characteristics.

\section{Main text}

\section{Materials and methods}

Formalin-fixed paraffin-embedded (FFPE) tissue samples from patients diagnosed in 2012-2019 with primary cutaneous nodular melanoma were collected from the archives of the Department of Anatomical Pathology, Dr. Sardjito Hospital, which is the main cancer referral center in Yogyakarta, Indonesia. Cases with prior chemotherapy or radiotherapy, incomplete clinical data, and degraded specimens were excluded. Thirty-one samples were analyzed in this retrospective cohort study, and all patients were of Javanese ethnicity.

RNA was extracted from FFPE tissues using Gene$\mathrm{All}^{\circledR}{ }^{\circledR}$ Ribospin ${ }^{\mathrm{TM}}$ II (GeneAll Biotechnology, Seoul, South Korea). Real-time polymerase chain reaction (RT-PCR) for $P D-L 1$ and NKG2A expression quantification was conducted using AccuPower ${ }^{\circledR}$ GreenStar ${ }^{\text {TM }}$ RT-qPCR PreMix on an Exicycler ${ }^{\mathrm{TM}} 96$ (Bioneer Corp., Daejeon, South Korea) with primer pairs and thermocycler conditions as previously described by Vassilakopoulou et al. and Meckawy et al. $[13,14]$. The expressions of $P D-L 1$ and NKG2A were calculated from the quantification cycle $(\mathrm{Cq}) \mathrm{val}-$ ues of the gene targets and normalized against GAPDH as an internal control. Subsequent normalization was performed using the $\Delta \Delta \mathrm{Cq}$ values of RNA derived from healthy skin tissues. Age, sex, tumor location, Breslow thickness, greatest diameter, lymph node involvement, and stage were retrieved from medical records. Pathological data, including the presence of necrosis, lymphovascular invasion, tumor-infiltrating lymphocytes (TILs), and mitotic index, were obtained from hematoxylineosin and Ki-67 IHC stained slides. Survival status (living or deceased) was determined through telephone calls at the point of follow-up of the study (until April 2020).

Samples were classified as normoregulated if the expression was lower than or equal to the mean of the $P D-L 1$ and NKG2A levels; conversely, samples were classified as upregulated if the expression was above the mean. Comparison of mRNA level averages based on categorical clinicopathologic characteristics was performed using Mann-Whitney U tests. Spearman correlation was used to analyze associations between the expression of $P D-L 1$ and NKG2A and continuous clinicopathologic features. Kaplan-Meier analysis and log-rank tests with Cox regression were used to determine hazard ratios (HRs) for survival analysis. To minimize the effect from Breslow thickness, two samples was excluded from survival analysis due to depth of less than $4 \mathrm{~mm}$.

\section{Results}

The characteristics of the subjects are presented in Table 1. Most tumors were located on the extremities (70.97\%) and thicker than $4 \mathrm{~mm}$ (93.55\%). Necrosis and TILs were present in $74.19 \%$ of the samples, respectively. The clinical stages were evenly distributed among stages II (29.03\%), III (35.48\%), and IV (35.48\%).

The expression of $P D-L 1$ and NKG2A was not significantly associated with the patients' clinicopathologic characteristics (Additional file 1: Table S1). Spearman correlation showed that NKG2A and PD-L1 mRNA levels were strongly correlated (Additional file 2: Table S2).

In the Cox univariate regression analysis, higher stage, upregulated $P D-L 1$, and upregulated $N K G 2 A$ were related to higher risks of death, with respective HR of $1.080(p=0.763), 2.429(p=0.101)$, and $1.011(p=0.981)$ (Additional file 3: Table S3). In multivariate analysis, the HR for PD-L1 increased to $3.488(p=0.066)$, while the other HRs decreased to $1.017(p=0.951)$ for stage and $0.590(p=0.391)$ for NKG2A upregulation. However, the differences were not statistically significant.

Patients with normoregulated $P D-L 1$ expression had longer median survival time ( $28.945 \pm 4.126$ months) compared with upregulated expressers $(15.800 \pm 2.345$ months; $p=0.086)$ (Fig. 1). Similar findings were observed for the normoregulated (26.470 \pm 3.854 months) and upregulated NKG2A subjects (25.943 \pm 7.415 months; $p=0.981)$ (Fig. 2). However, both differences were not significant statistically. Presence of TILs did not affect the survival curves 
Table 1 Clinicopathologic characteristics of the subjects

\begin{tabular}{|c|c|}
\hline Age (years), mean $\pm S D$ & $61.68 \pm 16.54$ \\
\hline \multicolumn{2}{|l|}{ Sex, n (\%) } \\
\hline Male & $8(25.81)$ \\
\hline Female & $23(74.19)$ \\
\hline \multicolumn{2}{|l|}{ Tumor location, n (\%) } \\
\hline Trunk & $2(6.45)$ \\
\hline Head and neck & $7(22.58)$ \\
\hline Extremity & $22(70.97)$ \\
\hline \multicolumn{2}{|l|}{ Lymph node metastases, n (\%) } \\
\hline Present & $21(67.74)$ \\
\hline Absent & $10(32.26)$ \\
\hline \multicolumn{2}{|l|}{ Breslow thickness } \\
\hline$\leq 4.00 \mathrm{~mm}$ & $2(6.45)$ \\
\hline$>4.00 \mathrm{~mm}$ & $29(93.55)$ \\
\hline \multicolumn{2}{|l|}{ Necrosis, n (\%) } \\
\hline Present & $23(74.19)$ \\
\hline Absent & $8(25.81)$ \\
\hline Tumor diameter $(\mathrm{mm})$, mean $\pm \mathrm{SD}$ & $30.00 \pm 24.09$ \\
\hline \multicolumn{2}{|l|}{ Ulceration } \\
\hline Present & $16(51.61)$ \\
\hline Absent & $15(48.39)$ \\
\hline \multicolumn{2}{|l|}{ Tumor-infiltrating lymphocytes } \\
\hline Present (brisk and non-brisk) & $23(74.19)$ \\
\hline Absent & $8(25.81)$ \\
\hline \multicolumn{2}{|l|}{ Clinical stage } \\
\hline I & $0(0.00)$ \\
\hline$\|$ & $9(29.03)$ \\
\hline III & $11(35.48)$ \\
\hline IV & $11(35.48)$ \\
\hline \multicolumn{2}{|l|}{ Survival status } \\
\hline Alive & $7(22.58)$ \\
\hline Deceased & $24(77.42)$ \\
\hline Overall survival (months), mean $\pm S D$ & $22.84 \pm 15.75$ \\
\hline
\end{tabular}

SD: standard deviation

significantly $(p=0.422)$ (Additional file 4 : Fig. S1). The survival curves of the upregulated and normoregulated groups for $P D-L 1$ and NKG2A did not differ significantly when divided based on the presence of TILs (Additional file 5: Fig. S2 and Additional file 6: Fig. S3).

\section{Discussion}

In this study, we compared the clinicopathologic characteristics and overall survival of Indonesian primary nodular melanoma cases with different mRNA levels of $P D-L 1$ and NKG2A. Two important findings were observed: (1) cases with $P D-L 1$ overexpression tended to have lower survival rates and (2) $P D-L 1$ and $N K G 2 A$ levels were strongly correlated.
Melanoma patients with upregulated $P D-L 1$ tended to have lower overall survival, with an approximately two to threefold higher HR compared to normal expressers. This trend was not proven statistically significant, possibly due to the small sample size. However, the increase in the $\mathrm{HR}$ for $P D-L 1$ upregulation in multivariate regression suggests this trend may exist in the overall population of melanoma patients in Indonesia.

The $P D-L 1$ molecule interacts with $P D-1$ receptors on $\mathrm{T}$ cells, causing anergy, exhaustion, and apoptosis [15]. Melanoma cells can thus evade the immune system by increasing their $P D-L 1$ expression. Melanoma cell line studies show that cells with upregulated $P D-L 1$ demonstrate highly invasive and aggressive behavior [16]. In a study on melanoma patients treated with surgery and dacarbazine, patients with positive $P D-L 1$ on IHC staining had lower median survival time compared with the subgroup with negative or indeterminate $P D-L 1$ status (9.7 months vs. 11.6 months) [17].

Our results seem to oppose those of Gupta et al., who observed that higher $P D-L 1$ mRNA levels reflect better prognoses for melanoma patients treated with anti- $P D-1$ agents [12]. This disparity may have stemmed from differences in the treatment regimen. Patients with high expression of $P D-L 1$ respond well to anti-PD-1 antibodies, hence the increase in survival [5]. The results suggest that $P D-L 1$ expression is a negative prognostic factor in patients with melanoma treated with conventional chemotherapy. However, when treated with anti-PD-1 antibodies, patients with high levels of $P D-L 1$ respond well and have good outcomes. Therefore, the choice of therapy also affects the performance of $P D-L 1$ as a prognostic factor.

Tumor cells can express $P D-L 1$ independently (called constitutive expression) or in response to TILs (reactive expression). TILs can secrete interferon-gamma, which induces the expression of $P D-L 1$ in tumor cells [15]. When $P D-L 1$ expression is accompanied by the presence of TILs, the expression is likely reactive, and vice versa. Due to the strongly positive correlation between $P D-L 1$ and TILs, studies report that most melanomas express $P D-L 1$ reactively [18]. However, in our study, the proportion of upregulated PD-L1 among the cases without TILs was higher (37.5\%) than the cases with TILs (25\%). The groups without TILs also had higher average PD-L1 mRNA levels than the group with TILs. This finding suggests that the cases with upregulated $P D-L 1$ in our study are likely constitutive expressers.

These two modes of $P D-L 1$ expression may have different prognostic implications. When previous studies divided patients based on $P D-L 1$ expression and the presence of TILs, patients with constitutive $P D-L 1$ expression without lymphocyte infiltrates showed the 


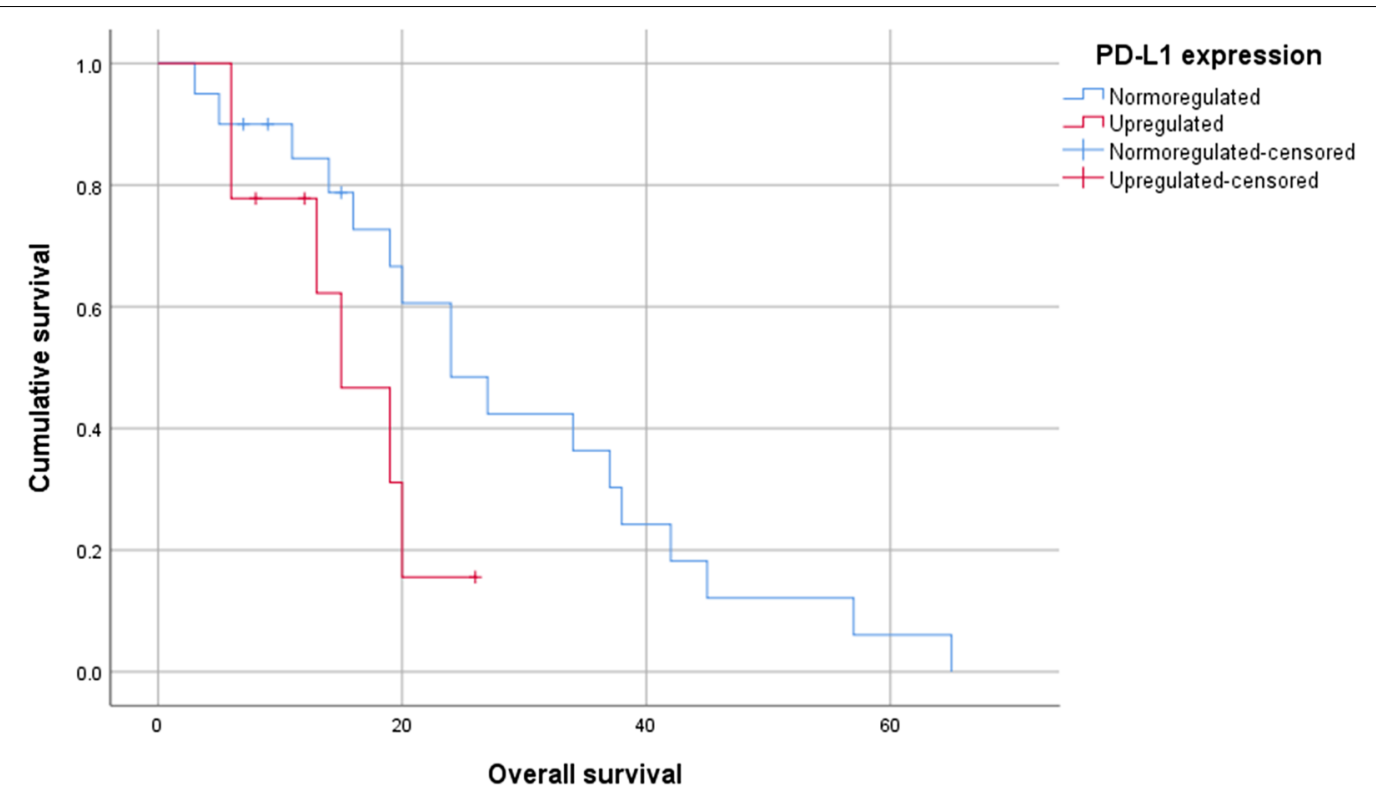

Fig. 1 Kaplan-Meier survival curves comparing the survival of patients with primary nodular melanoma with upregulated or normoregulated PD-L1 expression $(p=0.086)$

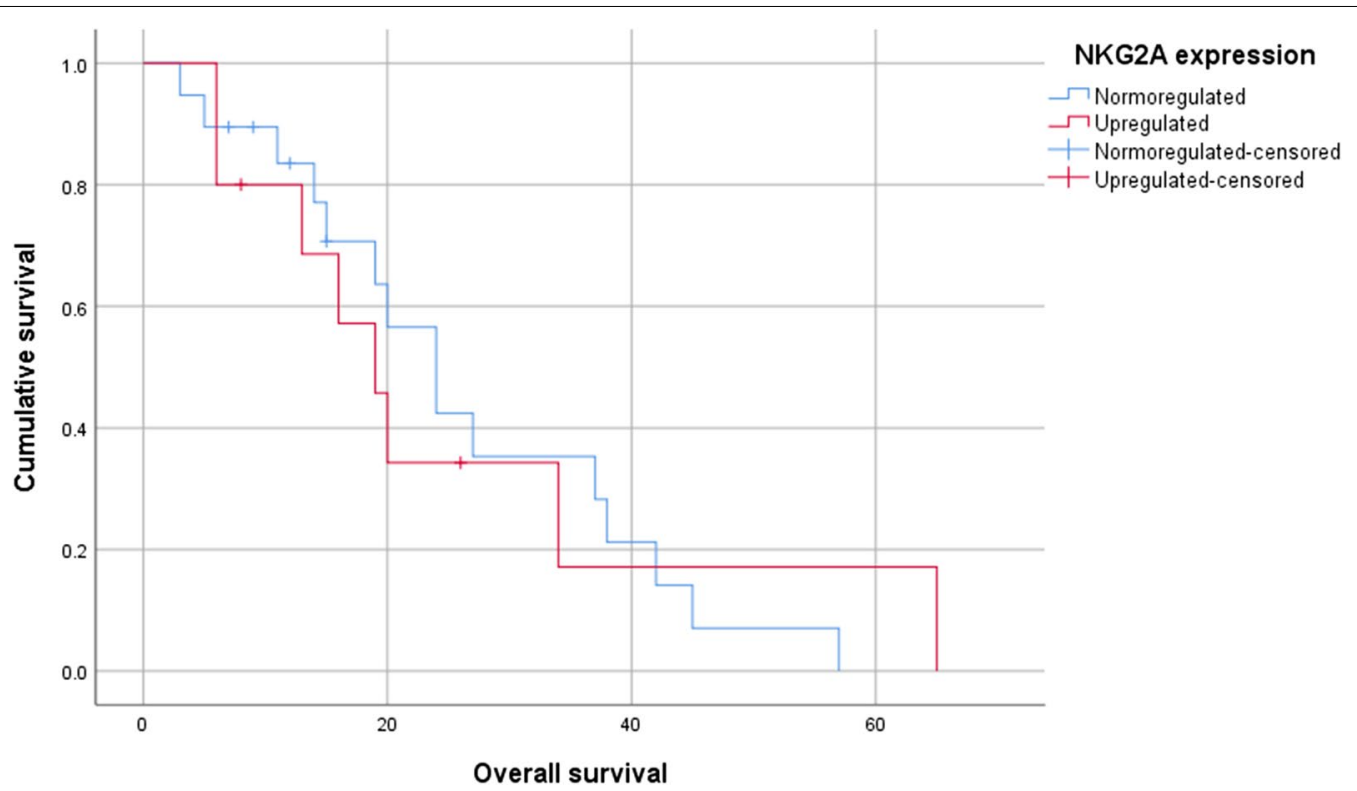

Fig. 2 Kaplan-Meier survival curves comparing the survival of patients with primary nodular melanoma with upregulated or normoregulated NKG2A expression $(p=0.981)$

poorest outcomes, followed by reactive $P D-L 1$ expressers, those with $P D-L 1(-)$ without TILs, and, finally, those with $P D-L 1(-)$ and TILs $[16,19]$. In our results, both groups with upregulated $P D-L 1$ showed poorer prognoses than the groups with normoregulated $P D$ L1. However, survival did not differ significantly when the cases were divided further based on TIL status, likely because none of the patients were treated using immunotherapy, in which the presence of TILs predicts improved response [20]. Another factor that could explain this lack of significance is the limited sample size.

Upregulated NKG2A mRNA did not appear to affect survival, given the highly insignificant results in 
univariate and multivariate regression analysis. NKG2A is an inhibitory receptor found on NK cells [21]. Cancer cells can attempt to evade the immune system by upregulating HLA self-molecules that activate NKG2A receptors and impair the function of NK cells. Trials in mouse models indicate that monalizumab is ineffective as a single therapy but highly effective when used together with other immunotherapy agents that promote activated TILs, such as anti-PD-1 or cancer vaccines [22]. One escape strategy used by cells to escape cytotoxic TILs is downregulation of MHC I expression, which renders them targets for NK cells [23]. This finding may explain the role of anti-NKG2A as an adjunct treatment for other immunotherapies. Our results reinforce the idea that $N K G 2 A$ may not be an independent therapeutic target and prognostic factor but may play a role in combination therapy.

The mRNA expressions of $N K G 2 A$ and $P D-L 1$ were strongly correlated. This finding indicates that tumors with high $P D-L 1$ expression would also likely express $N K G 2 A$ strongly and, thus, respond well to anti-NKG2A agents. When $N K G 2 A$ expression was combined with the TIL parameter, the distribution of survival curves obtained resembled the curves for $P D-L 1$ combined with TILs. NKG2A upregulation with and without the presence of TILs may have different pathogeneses and prognostic implications, like $P D-L 1$.

The lack of correlation between the expression of $N K G 2 A$ and $P D-L 1$ and clinicopathologic characteristics in this work resembles the findings of several previous studies $[6,18]$. The small sample size of this study may have contributed to the low statistical significance found.

The findings of this study must be interpreted with caution especially due to the small sample size. However, our results support the findings of several studies that show that mRNA profiles may serve as a prognostic factor in melanoma cases [12, 24]. Further research and clinical trials are needed to ascertain the roles of $P D-L 1$ and $N K G 2 A$ in the prognosis and therapy of Asian patients who have not previously received immune checkpoint inhibitors.

\section{Conclusions}

We investigated the correlations between the mRNA levels of $P D-L 1$ and NKG2A with clinicopathologic characteristics and survival in primary nodular melanoma patients in Yogyakarta, Indonesia. Patients with upregulated $P D-L 1$ expression had shorter median overall survival, although insignificant statistically. $P D-L 1$ and $N K G 2 A$ mRNA levels were positively correlated.

Our findings suggest that the therapy regimen and presence of TILs may affect the prognostic role of $P D-L 1$ expression. NKG2A was not proven to be an independent predictive factor but may serve as an adjunct target for therapy. The strong correlation between $P D-L 1$ and $N K G 2 A$ suggests that anti-PD1 and anti-NKG2A agents may be effective in patients with $P D-L 1$ upregulation. Studies with larger subject groups are needed to confirm the patterns of $P D-L 1$ expression in Asian cases.

\section{Limitations}

Our study was limited by its small sample size and homogenous ethnic population. Results among diverse Indonesian and Asian populations may differ. TILs examination did not discriminate between lymphocyte subtypes.

\section{Abbreviations}

CD: Cluster of differentiation; Cq: Quantification cycle; DNA: Deoxyribonucleic acid; FFPE: Formalin-fixed paraffin-embedded; GADPH: Glyceraldehyde 3-phosphate dehydrogenase; HLA: Human leukocyte antigen; IHC: Immunohistochemistry; MHC: Major histocompatibility complex; mRNA: Messenger ribonucleic acid; NK: Natural killer; NKG2A: Natural killer group 2A; PD-1:

Programmed death-1; PD-L1: Programmed death-ligand 1; RT-PCR: Real-time polymerase chain reaction; TILS: Tumor-infiltrating lymphocytes.

\section{Supplementary Information}

The online version contains supplementary material available at https://doi. org/10.1186/s13104-021-05623-7.

Additional file 1: Table S1. Comparison of $P D-L 1$ and NKG2A expression levels based on clinicopathologic characteristics and overall survival.

Additional file 2: Table S2. Spearman correlations between continuous variables.

Additional file 3: Table S3. Univariate and multivariate Cox regression results for stage, $P D-L 1$ upregulation, and NKG2A upregulation.

Additional file 4: Fig. S1. Kaplan-Meier survival curves comparing the survival of patients with primary nodular melanoma with or without TILs.

Additional file 5: Fig. S2. Kaplan-Meier survival curves comparing the survival of patients with primary nodular melanoma based on PD-L1 expression and the presence of TILS.

Additional file 6: Fig. S3. Kaplan-Meier survival curves comparing the survival of patients with primary nodular melanoma based on NKG2A expression and the presence of TILs.

\section{Acknowledgements}

We would like to thank Nur Eka Wiraditya (Department of Anatomical Pathology, Faculty of Medicine, Public Health and Nursing, Universitas Gadjah Mada, Yogyakarta, Indonesia) for assisting us with the laboratory procedures in this study.

\section{Authors' contributions}

RDS designed the study and performed the analysis. HTR, MFP, SLA, KS, and TA wrote the manuscript. YI and MRR contributed to the data collection. All authors read and approved the final manuscript.

\section{Funding}

This study was funded by Universitas Gadjah Mada through Program Rekognisi Tugas Akhir 2020 (2607/UN1/DITLIT/DIT-LIT/PT/2020). Laboratory reagents and consumables constituted most of the expenditures. Publication costs were 
covered by the Faculty of Medicine, Public Health and Nursing, Universitas Gadjah Mada.

\section{Availability of data and materials}

This submission contains all of the data analyzed during the study. Unprocessed data can be requested from the corresponding author.

\section{Declarations}

\section{Ethics approval and consent to participate}

This study complied with the Declaration of Helsinki and the Belmont Report. The protocol for this study was approved by the Medical and Health Research Ethics Committee of the Faculty of Medicine, Public Health, and Nursing, Universitas Gadjah Mada (KE/FK/0599/EC/2020). The study was conducted with formal permission from the appropriate hospital officials after explaining the research objectives and procedures. Patients were informed preoperatively of the use of data and tissue samples for research. All patients have consented in written forms.

\section{Consent for publication}

Not applicable.

\section{Competing interests}

The authors declare that they have no competing interests.

\section{Author details}

'Department of Surgery, Faculty of Medicine, Public Health and Nursing, Universitas Gadjah Mada/Dr. Sardjito Hospital, Sleman, Yogyakarta, Indonesia. ${ }^{2}$ Department of Anatomical Pathology, Faculty of Medicine, Public Health and Nursing, Universitas Gadjah Mada/Dr. Sardjito Hospital, Sleman, Yogyakarta, Indonesia.

Received: 16 December 2020 Accepted: 19 May 2021

Published online: 28 May 2021

\section{References}

1. International Agency for Reserch on Cancer. GLOBOCAN fact sheet: melanoma of skin. Global Cancer Observatory. 2018. http://globocan.iarc. fr/old/bar_sex_site.asp?selection $=16120 \&$ title=Melanoma+of + skin\& statistic $=2 \&$ populations $=6 \&$ window $=1 \& \mathrm{grid}=1 \&$ color $1=5 \&$ color $1 \mathrm{e}=\&$ color $2=4 \&$ color $2 e=\&$ submit $=$ Execute.

2. International Agency for Research on Cancer. Estimated number of incident cases from 2018 to 2040: Melanoma of skin. Cancer Tomorrow. 2018. https://gco.iarc.fr/tomorrow/graphic-line?type=0\&type_sex=0\&mode $=$ population \&sex=0\&populations $=900 \&$ cancers $=16 \&$ age_group $=$ value $\&$ apc_male $=0 \& a p c \_$female $=0 \&$ single_unit $=500000 \&$ print $=0$.

3. Domingues B, Lopes JM, Soares P, Pópulo H. Melanoma treatment in review. ImmunoTargets Ther. 2018;7:35-49.

4. Van Hall T, André P, Horowitz A, Ruan DF, Borst L, Zerbib R, et al. Monalizumab: inhibiting the novel immune checkpoint NKG2A. J Immunother Cancer. 2019;7(1):263.

5. Abdel-Rahman OPD. PD-L1 expression and outcome of advanced melanoma patients treated with anti-PD-1/PD-L1 agents: a meta-analysis. Immunotherapy. 2016;8(9):1081-9.

6. Yang J, Dong M, Shui Y, Zhang Y, Zhang Z, Mi Y, et al. A pooled analysis of the prognostic value of PD-L1 in melanoma: evidence from 1062 patients. Cancer Cell Int. 2020;20(1):96. https://doi.org/10.1186/ s12935-020-01187-x.

7. Patel SP, Kurzrock R. PD-L1 expression as a predictive biomarker in cancer immunotherapy. Mol Cancer Ther. 2015;14(4):847-56.

8. Lee HH, Wang YN, Xia W, Chen CH, Rau KM, Ye L, et al. Removal of N-linked glycosylation enhances PD-L1 detection and predicts anti-PD-1/PD-L1 therapeutic efficacy. Cancer Cell Int. 2019;36(2):168-178.e4.
9. Tang Y, Xie C, Zhang Y, Qin Y, Zhang W. Overexpression of mRNAdecapping enzyme 1a predicts disease-specific survival in malignant melanoma. Melanoma Res. 2018;28(1):30-6.

10. Hoffmann F, Zarbl R, Niebel D, Sirokay J, Fröhlich A, Posch C, et al. Prognostic and predictive value of PD-L2 DNA methylation and mRNA expression in melanoma. Clin Epigenet. 2020;12(1):94.

11. Wan X, Liu R, Li Z. The prognostic value of HRAS mRNA expression in cutaneous melanoma. BioMed Res Int. 2017;2017:5356737.

12. Gupta S, McCann L, Chan YGY, Lai EW, Wei W, Wong PF, et al. Closed system RT-qPCR as a potential companion diagnostic test for immunotherapy outcome in metastatic melanoma. J Immunother Cancer. 2019:7(1):254

13. Vassilakopoulou M, Avgeris M, Velcheti V, Kotoula V, Rampias T, Chatzopoulos K, et al. Evaluation of PD-L1 expression and associated tumor-infiltrating lymphocytes in laryngeal squamous cell carcinoma. Clin Cancer Res. 2016;22(3):704-13.

14. Meckawy GR, Mohamed AM, Zaki WK, Khattab MA, Amin MM, EIDeeb MA, et al. Natural killer NKG2A and NKG2D in patients with colorectal cancer. J Gastrointest Oncol. 2019;10(2):218-25.

15. Taube JM, Anders RA, Young GD, Xu H, Sharma R, McMiller TL, et al. Colocalization of inflammatory response with $\mathrm{B} 7-\mathrm{H} 1$ expression in human melanocytic lesions supports an adaptive resistance mechanism of immune escape. Sci Transl Med. 2012;4(127):127ra37.

16. Audrito V, Serra S, Stingi A, Orso F, Gaudino F, Bologna C, et al. PD-L1 up-regulation in melanoma increases disease aggressiveness and is mediated through miR-17-5p. Oncotarget. 2017;8(9):15894-911.

17. Robert C, Long GV, Brady B, Dutriaux C, Di Giacomo AM, Mortier L, et al. Five-year outcomes with nivolumab in patients with wild-type BRAF advanced melanoma. J Clin Oncol. 2020;38(33):3937-46.

18. Obeid JM, Erdag G, Smolkin ME, Deacon DH, Patterson JW, Chen L, et al. PD-L1, PD-L2 and PD-1 expression in metastatic melanoma: correlation with tumor-infiltrating immune cells and clinical outcome. Oncoimmunology. 2016;5(11):e1235107. https://doi.org/10.1080/2162402X.2016. 1235107.

19. Massi D, Brusa D, Merelli B, Falcone C, Xue G, Carobbio A, et al. The status of PD-L1 and tumor-infiltrating immune cells predict resistance and poor prognosis in BRAFi-treated melanoma patients harboring mutant BRAFV600. Ann Oncol. 2015;26(9):1980-7. https://doi.org/10.1093/ annonc/mdv255.

20. Badalamenti G, Fanale D, Incorvaia L, Barraco N, Listì A, Maragliano R, et al. Role of tumor-infiltrating lymphocytes in patients with solid tumors: can a drop dig a stone? Cell Immunol. 2017;2019(343):103753. https://doi. org/10.1016/j.cellimm.2018.01.013.

21. Zaghi E, Calvi M, Marcenaro E, Mavilio D, Di Vito C. Targeting NKG2A to elucidate natural killer cell ontogenesis and to develop novel immune-therapeutic strategies in cancer therapy. J Leukoc Biol. 2019;105(6):1243-51.

22. Borst $L$, van der Burg $S H$, van Hall T. The NKG2A-HLA-E axis as a novel checkpoint in the tumor microenvironment. Clin Cancer Res. 2020;26(21):5549-56.

23. Lee H, Quek C, Silva I, Tasker A, Batten M, Rizos H, et al. Integrated molecular and immunophenotypic analysis of NK cells in anti-PD-1 treated metastatic melanoma patients. Oncoimmunology. 2019;8(2):e1537581. https://doi.org/10.1080/2162402X.2018.1537581.

24. Jayawardana K, Schramm SJ, Haydu L, Thompson JF, Scolyer RA, Mann GJ, et al. Determination of prognosis in metastatic melanoma through integration of clinico-pathologic, mutation, mRNA, microRNA, and protein information. Int J Cancer. 2015;136(4):863-74.

\section{Publisher's Note}

Springer Nature remains neutral with regard to jurisdictional claims in published maps and institutional affiliations. 